\title{
Functional dysphonia: strategies to improve patient outcomes
}

This article was published in the following Dove Press journal:

Patient Related Outcome Measures

I December 2015

Number of times this article has been viewed

\section{Mara Behlau \\ Glaucya Madazio \\ Gisele Oliveira}

Voice Department, Centro de Estudos da Voz - CEV, São Paulo, Brazil

Correspondence: Mara Behlau Voice Department, Centro de Estudos da Voz - CEV, Rua Machado Bittencourt 36I, São Paulo 04044-00I, Brazil

Tel +55 II 5575 I7। I0

Fax +55 । 5575 ।7। 0

Email mbehlau@uol.com.br
Abstract: Functional dysphonia (FD) refers to a voice problem in the absence of a physical condition. It is a multifaceted voice disorder. There is no consensus with regard to its definition and inclusion criteria for diagnosis. FD has many predisposing and precipitating factors, which may include genetic susceptibility, psychological traits, and the vocal behavior itself. The assessment of voice disorders should be multidimensional. In addition to the clinical examination, auditory-perceptual, acoustic, and self-assessment analyses are very important. Self-assessment was introduced in the field of voice 25 years ago and has produced a major impact in the clinical and scientific scenario. The choice of treatment for FD is vocal rehabilitation by means of direct therapy; however, compliance has been an issue, except for cases of functional aphonia or when an intensive training is administered. Nevertheless, there are currently no controlled studies that have explored the different options of treatment regimens for these patients. Strategies to improve patient outcome involve proper multidisciplinary diagnosis in order to exclude neurological and psychiatric disorders, careful voice documentation with quantitative measurement and qualitative description of the vocal deviation for comparison after treatment, acoustic evaluation to gather data on the mechanism involved in voice production, self-assessment questionnaires to map the impact of the voice problem on the basis of the patient's perspective, referral to psychological evaluation in cases of suspected clinical anxiety and/or depression, identification of dysfunctional coping strategies, self-regulation data to assist patients with their vocal load, and direct and intensive vocal rehabilitation to reduce psychological resistance and to reassure patient's recovery. An international multicentric effort, involving a large population of voicedisordered patients with no physical pathology, could produce enough data for achieving a consensus regarding this complex problem.

Keywords: voice, dysphonia, evaluation, patient-related outcomes, quality of life, voice handicap

\section{Multidisciplinary diagnosis}

The human voice is a complex phenomenon. An individual's vocal quality depends on anatomofunctional features, psychological traits, and social and professional aspects. Voice disorders, also called dysphonias, occur in 3\%-9\% of the population. ${ }^{1}$ Dysphonia is any deviation that may restrain the natural voice production ${ }^{2}$ and that impacts the quality of life. The American Academy of Otolaryngology - Head and Neck Surgery Foundation published a clinical guideline about "hoarseness". In the guideline, dysphonia is characterized as any "deviation in the vocal quality, pitch, loudness, and vocal effort that affect communication or produces a negative impact on the voice-related quality of life". In other words, it is an individual's reduction of the self-perceived physical, emotional, social, or economic status due to a voice problem. ${ }^{1}$ 
Dysphonia can be etiologically and traditionally classified into two main broad categories: organic and functional types. ${ }^{2}$ Organic dysphonias are the consequences of aspects nonrelated to the use of voice, such as gastroesophageal reflux, vocal fold paralysis, and systemic diseases, eg, Parkinson's and amyotrophic lateral sclerosis. Functional dysphonias (FDs) are the results of phonotraumatic events (abusive behaviors or voice misuse), poor vocal technique, and/or muscle imbalance, with or without psychoemotional involvement.

FD is sometimes referred to as behavioral dysphonias, since the deviated vocal gesture is at the core of this disturbance. The label "functional voice disorders" has been at the center of scientific debates since the 1960s. Since then, there has been no consensus regarding its usage and concept. Particularly in the last 2 decades, American authors either avoid using the term FD or replace it with muscle tension dysphonia (MTD). ${ }^{46}$ When MTD is used as a synonym of FD, it can be differentiated into two types ${ }^{6}$ : primary and secondary MTD. Primary MTD is a voice disorder in which excessive atypical or abnormal laryngeal movements are observed during phonation in the absence of any of the following: organic pathology, and psychogenic or neurologic etiology. Secondary MTD is a voice disorder in which excessive compensatory atypical or abnormal laryngeal movements are seen during phonation in the presence of organic vocal pathology, psychogenic, or neurologic problem, originated as a response to the primary etiology. In addition to MTD, the other common terms used to refer to FD are as follows: psychogenic dysphonias, hyper-, and hypo-FDs, with differentiations according to the authors. ${ }^{7}$ Particularly for MTD cases, the situation is so complex, that three etiological subgroups can be recognized: ${ }^{8}$ 1) psychological and/or personality factors, 2) vocal misuse and abuse, and 3) compensation for the underlying disease.

FD can manifest itself with different voice qualities. There is not a single vocal pattern, which characterizes these patients' voice disorder. Weak voice, strained sound, hoarse or breathy vocal qualities, and whispering phonation, as well as lack of vocal efficacy, vocal fatigue, and kinesthetic symptoms (effort during speech) can be seen. In cases of total loss of voice, vegetative sounds (coughing, throat clearing, and laughing) are frequently preserved. ${ }^{9}$ For some authors, MTD should be regarded more as a speech problem than a voice disorder. ${ }^{4,10}$

Vocal behavior is at the center of FD; however, the ethiopathogenesis is complex and can involve anatomofunctional predispositions, such as small glottic proportions ${ }^{11}$ and psychological traits. ${ }^{5,12,13}$ A deviated vocal behavior with inadequate muscle activity can produce benign mass lesions, such as vocal nodules and polyps. These cases are sometimes called organofunctional or behaviorally based dysphonias with benign mass lesions. ${ }^{14}$

In order to suggest strategies to improve the outcomes for the FD patient, we need to understand certain aspects of its diagnosis and treatment.

\section{Voice documentation with multidimensional descriptions}

The assessment of a patient with a voice problem is multidimensional and will usually include the following procedures: 1) visual laryngeal examination (via nasal or oral endoscopy, to visualize the vocal folds and detect lesions and/or problems with muscle activity); 2) auditory-perceptual analysis of voice quality (to identify the degree and type of deviation); 3) acoustic/aerodynamic measures (to quantify different aspects related to the fundamental frequency, noise parameters, and maximum phonation time); and 4) self-assessment tools (to identify the perception of the patient with regard to the impact of the voice disorder on his/her life). Additionally, the identification of behavioral aspects related to the use of voice must be assessed for a thorough diagnosis. These aspects need to be carefully considered, ideally by collaboration between the physician and the speech-language pathologist/ voice specialist to properly deal with clinical requirements. A combined evaluation by these two professionals is the best option for improving diagnostic precision and patient's adherence to treatment, as well as to reduce health costs. ${ }^{15}$

The clinical evaluation of voice that includes multiple procedures is a common practice in the field; however, during the last few decades, instruments that measure the patient's experience of living with a voice problem hold a special place in the armamentarium of assessment. ${ }^{16-21}$

The multidimensional voice evaluation can be grouped into two main categories: clinician-centered perspective and patient-centered perspective.

\section{Clinician-centered perspective}

This perspective usually includes laryngeal examination, auditory-perceptual analysis, and some acoustic/aerodynamic measurements.

\section{Laryngeal examination}

Laryngeal analysis is the focus of the medical examination in the presence of a voice symptom. Laryngeal structures, particularly the vocal folds, are assessed during breathing 
and phonation. Laryngoscopy is the minimal examination required for voice diagnosis. ${ }^{1}$ Videolaryngostroboscopy (VLS) is a well-established procedure that analyzes the vocal folds' vibration and has become the routine examination method for voice problems. ${ }^{22}$ VLS is critical to evaluate a dysphonic patient and its use increases diagnostic accuracy in $68.3 \%$ of cases of hoarseness. ${ }^{23}$ In case of FD, special attention is given to the glottal closure, vocal fold vibration, mucosal wave, and supraglottic activity. Even though, VLS is a useful tool for voice evaluation, it should not be the sole method used for diagnostic purposes, since there is no relationship between the clinical course and changes in stroboscopic data. ${ }^{24}$ Recent technical advances, such as highspeed cameras, are more sensitive and offer more detailed information about phonatory function. However, they are expensive and there are many methodological challenges to overcome (such as excessive data and time-consuming analysis) before proposing it as a clinical tool for the evaluation of patients with voice problems. ${ }^{23}$ An external examination (palpation) of laryngeal extrinsic muscles, neck, mandible and observation of facial gestures during speech can add important information particularly for the cases of FD due to MTD, ${ }^{4,10}$ even if there is reduced information on the validity of these assessments. ${ }^{10}$

\section{Auditory-perceptual analysis}

Voice is a fundamentally perceptual phenomenon, thus perceptual evaluation is a strong candidate for the gold standard assessment of patients with voice disorders. ${ }^{25}$ Although widely used for diagnosis, treatment outcome, follow-ups, and dismissal, reliability problems were pointed out since early studies were performed. ${ }^{26-28}$ This reliability issue is even considered as a noncontrolled effect of the human auditory processing nature. ${ }^{29}$ Some variables, such as type of stimuli, presentation context, personal and professional experiences, and cultural influences, ${ }^{26,27,29}$ have been repeatedly highlighted as interfering factors. These aspects can be minimized by standardizing the assessment protocol and training of listeners, but it does not solve the problem for FD cases since patients usually show instability in their voices and mixed components of roughness, breathiness, and strain. The overall degree of vocal deviation, which reflects the total amount of abnormality, has been used to reduce the reliability problem. ${ }^{14}$

\section{Acoustic/aerodynamic measurements}

Acoustic analysis aims to measure different parameters of the voice signal. It is considered as a noninvasive quantitative assessment of vocal quality, objective in nature, or at least, semiobjective since there is a lot of human interaction. Humans are involved in the process of developing the software, selecting the algorithm, recording, storing, and analyzing the signal.

It is important to highlight that the acoustic analysis is only a part of the voice evaluation process. Its efficiency depends essentially on the clinician's ability to integrate the findings of perceptual, acoustic, and laryngeal imaging analyses. Among the suitability of the acoustic analysis, the main aspects are to facilitate the comprehension of voice production, generate normative data, produce vocal documentation, monitor treatment outcome, follow-up the voice development, and early detection of voice problems. The acoustic analysis represents the objective portion of the voice evaluation process. Due to its objective nature, it allows the transformation of an abstract construct into a concrete reality.

Acoustic analysis gained popularity in the 1990s due to the development of inexpensive computer-based programs that allowed the average clinician to obtain data previously limited to university and hospital voice laboratories. The use of these measures in isolation is controversial. ${ }^{30}$ However, some important information about the sound composition and production can be collected and compared to the perceptual data. Since voice has harmonic and noise components, parameters are related to each of these aspects. Fundamental frequency measurements, at least their extraction, are shown to be robust parameters. The same is not true for perturbation analysis, such as jitter and shimmer. ${ }^{31}$ Noise parameters are considered clinically important, because the noisier the voice, the more distant it is from the normal vocal quality. Nonetheless, the reliability of noise measurements is related to many factors such as the overall deviation of vocal quality. ${ }^{32}$ The problem with this traditional acoustic analysis is that the quantification of the voice sample is based on the assumption that the signal is nearly periodic. However, this is not often the case for dysphonic voices, and quantification of these signals can be meaningless. ${ }^{32}$ A new approach has been recently described, the Cepstral Spectral Index of Dysphonia, ${ }^{33}$ which is a multivariate estimate of dysphonia severity. This measure seems to be a potentially robust tool for voice disorder identification; yet, its validity as an outcome measure has been limited to few studies. ${ }^{34}$

An alternative approach to dealing with dysphonic signals is to use nonlinear dynamical systems analysis ${ }^{35}$ but there are only few contributions in order to draw conclusions about its use for clinical purposes. ${ }^{36}$ 


\section{Patient-centered perspective - self- assessment questionnaires}

Voice-disordered quality of life is a disease-specific construct aimed to evaluate the impact of a voice problem in activity limitations and participation restrictions for a specific patient. The voice-disordered quality of life instruments were introduced in the late 1990s $\mathrm{s}^{16-18}$ and have been used for clinical diagnosis, to quantify the impact of a voice problem, to help adherence, to contribute in therapeutic management, to evaluate the patient's response to different treatments, and to screen large populations. These instruments not only showed good psychometric properties ${ }^{16-20,37-72}$ but also improved clinical care.

Self-assessment instruments reveal new information about the impact of dysphonia related to the quality of life that could not have been obtained by traditional approaches. Additionally, because there is a low correlation between the patient's perspective and the clinician's analysis, ${ }^{73,74}$ this type of investigation should be mandatory.

These instruments were initially developed mostly in English and were subsequently validated in several other languages in different countries (Table 1). The validated instruments showed comparable psychometric properties to their original versions. The worldwide spread of these instruments made us understand that there is a common universal trait for patients with voice problems. Physical (organic), functional (activity and participation), and emotional (socio-emotional) aspects are shared by all instruments.
Furthermore, specific scores of vocal activity limitation and participation restrictions can be obtained by using one instrument. ${ }^{19}$ Recently, cross-cultural differences of health, illness, and disability perception have also been documented in the area of voice. ${ }^{75,76}$

Self-assessment instruments have achieved rapid international popularity, which was not seen before with any other assessment approach, ${ }^{21}$ regardless of some methodological problems identified in their development process. ${ }^{42,77}$ Some reasons behind this popularity are, the global spread of the concept of health and disease by the World Health Oraganization, ${ }^{78}$ the excellent cost-benefit ratio of using questionnaires with no need of fancy equipment, the reduction of time for completing an objective self-evaluation (up to 5 minutes), its utility for voice diagnosis, with the possibility of quantifying and qualifying the impact of a voice disorder in a person's life; and the insights produced by simply answering a list of questions. A typical clinical observation from patients is that they say they were not aware of how much their voice problem caused them losses and negative implications until they answered the instrument.

No self-assessment instrument for voice disorders was specifically developed for the evaluation of a particular diagnostic category, including FD, except for the Voice Outcome Survey designed for patients with vocal fold paralysis. ${ }^{79}$ Nevertheless, these instruments are crucial because they reveal the subjective perception of negative impacts imposed by a voice problem. This information is unique and cannot

Table I Main self-assessment questionnaires for investigating the impact of FD, original country of development, and validation in other countries

\begin{tabular}{|c|c|c|}
\hline Name of instrument and mains aspects & Original country & Validation in other countries \\
\hline $\begin{array}{l}\text { Voice Handicap Index }(\mathrm{VHI}) \text { - 30-item questionnaire: total, } \\
\text { functional, organic, and emotional domains }\end{array}$ & USA $^{16}$ & $\begin{array}{l}\text { Germany, }{ }^{37} \text { Taiwan }{ }^{38} \text { Portugal, }{ }^{39} \text { France, }{ }^{40} \text { Poland, },{ }^{41} \\
\text { UK, }{ }^{42} \text { Germany, }{ }^{43} \text { the Netherlands, }{ }^{44} \text { Israel, },{ }^{45} \text { Scotland, }{ }^{46} \\
\text { Spain, }{ }^{47} \text { People's Republic of China, }{ }^{48} \text { Turkey, }{ }^{49} \\
\text { Sweden, }{ }^{50} \text { Brazil, }{ }^{51} \text { Italy, }{ }^{52} \text { Greece, }{ }^{53} \text { Saudi Arabia, }{ }^{54} \\
\text { Norway, }{ }^{55} \text { Japan, },{ }^{56} \text { Iran, },{ }^{57} \text { Croatia, }{ }^{58} \text { and Latvia }{ }^{59}\end{array}$ \\
\hline $\begin{array}{l}\text { Voice Handicap Index } 10(\mathrm{VHI}-10) \text { reduced version - I0-item } \\
\text { questionnaire: total score }\end{array}$ & USA $^{60}$ & $\begin{array}{l}\text { People's Republic of China, }{ }^{61} \text { Israel, }{ }^{62} \text { Brazil, }{ }^{63} \text { Spain, }{ }^{47} \\
\text { and Italy }{ }^{64}\end{array}$ \\
\hline $\begin{array}{l}\text { Voice-Related Quality of Life (V-RQOL) - I0-item } \\
\text { questionnaire: total score, physical functioning domain, and } \\
\text { social-emotional domain }\end{array}$ & USA $^{17}$ & Germany, ${ }^{65}$ Brazil $^{66}$ and India ${ }^{67}$ \\
\hline $\begin{array}{l}\text { Vocal Performance Questionnaire (VPQ) - I2-item } \\
\text { questionnaire: total score }\end{array}$ & $\mathrm{UK}^{18}$ & Brazil $^{68}$ \\
\hline $\begin{array}{l}\text { Voice Activity and Participation Profile (VAPP) - } 28 \text {-item } \\
\text { questionnaire, five aspects: total score and scores on self- } \\
\text { perceived severity of voice problem, effect on job, effect on } \\
\text { daily communication, effect on social communication, and effect } \\
\text { on emotion. Two extra-scores for activity and participation }\end{array}$ & $\begin{array}{l}\text { People's Republic of } \\
\text { China (Hong Kong) }\end{array}$ & Finland, ${ }^{69}$ Brazil, $^{70}$ and Italy ${ }^{71}$ \\
\hline $\begin{array}{l}\text { Voice Symptom Scale (VoiSS) - 30-item questionnaire: total, } \\
\text { impairment, emotional, and physical domains }\end{array}$ & $\mathrm{UK}^{20}$ & Brazil $^{72}$ \\
\hline
\end{tabular}

Abbreviation: FD, functional dysphonia. 
be obtained by either laryngeal examination or perceptual and acoustic analysis.

There are some differences among these instruments with regard to their conceptual development. Some of them are more focused on the perceived handicap (Voice Handicap Index), ${ }^{16}$ some on the quality of life (Voice-Related Quality of Life), ${ }^{17}$ some on the loss of vocal endurance (Voice Performance Questionnaire),$^{18}$ others on activity limitation and participation restriction (Voice Activity and Participation Profile),${ }^{19}$ and finally the other ones on combining disability and vocal symptoms (Voice Symptoms Scale). ${ }^{20}$

Studies on specific populations with voice problems need to be performed, particularly including professional voice users with functional voice problems, both artistic (singers and actors) and nonartistic (teachers, call center operators, and sales persons). The question with the artistic professional voice users is even more complex because some vocal deviations may be part of their signature voice. In this case, it can be difficult to differentiate between a stylistic choice and a behavioral problem. There have been some attempts to develop specific tools for this population, but currently no extensive data was derived. ${ }^{80,81}$ Professional voice users face different demands regarding voice quality and endurance to long periods of usage. It is plausible to consider that they may have a different sensibility to voice changes and also diverse ways of coping with the problem. A slight vocal problem that would do no harm to a nonprofessional voice user can severely impair the career of an elite vocal professional. Therefore, a similar degree of dysphonia for a nonprofessional voice user may be perceived differently. ${ }^{81}$

Finally, it is important to mention that voice is a cultural construct and vocal expression has been the mirror of cultural differences throughout mankind. There is a known relationship between voice, linguistic code, and cultural behavior, ${ }^{21}$ but unfortunately this has not yet been explored by voice clinicians. The exploration of cross-cultural differences began only recently in the voice area, both regarding self-assessment of the voice problem ${ }^{76}$ and the manifestation of specific disorders. ${ }^{82}$ When cultural modifications lead to qualitative changes in the voice, a voice disorder must be considered. ${ }^{21}$ This subject deserves proper attention to better suit specific aspects, particularly if we consider a globalized world with people living in different areas of the globe.

It is possible that self-assessment instruments capture a different aspect of the vocal function that cannot be derived from auditory-perceptual or acoustic analysis and laryngeal examination. It is also reasonable to say that some changes are seen because patients want to please clinicians and they know they will be assessed (Hawthorne effect). ${ }^{83}$ Therefore, clinicians must wisely use both clinician- and patient-oriented information for diagnosis and evaluation of treatment outcome.

\section{Psychological considerations: coping and self-regulation strategies}

Emotional issues can be clearly seen in some but not all cases Psychogenic factors seem to be more relevant in cases of total voice loss, functional aphonia, than in cases of variable voice deviations ${ }^{84}$ Using the personality traits construct, Roy et $\mathrm{al}^{5}$ compared patients among four diagnostic categories: FD, vocal fold nodules (both of them being behavioral-based cases), spasmodic dysphonia, and vocal fold paralysis (both of them being organic types). The findings made clear that the behavioral-based dysphonias have specific psychological traits. Individuals with FD were characterized as introverted, stress reactive, alienated, and unhappy. Patients with vocal nodules are socially dominant, stress-reactive, aggressive, and impulsive. Finally, the organic-based cases and subjects without voice disorders did not present any consistent personality features.

Patients with common voice disorders, including FD cases, assessed by the Hospital Anxiety and Depression Scale presented higher psychological distress. Stress and depression were more common in patients with MTD. In particular, females ${ }^{12}$ with FD usually present multiple psychosocial problems. ${ }^{86}$ It is not sufficient to look only at the vocal behavior; the other associated factors such as predisposition, precipitant, and maintenance factors must also be taken into consideration. ${ }^{86}$ Predisposition factors are genetic susceptibility, constitution of the individual, occupational susceptibility, prolonged stress, laryngeal inflammatory processes, history of sexual and/or physical abuse, and perfectionism. Precipitant factors are life events, vocal load, upper airway infections, and laryngeal inflammatory processes. Finally, the potential maintenance factors are probably prolonged stress and general fatigue ${ }^{84,85}$ The popular conception is that voice loss is a result of unexpressed emotion and has no scientific evidence. ${ }^{85}$

The specific role of psychogenic traits in the development of different categories of voice disorders as well as the interaction between predisposed and causal factors for FD is not completely understood. However, these factors have to be considered since FD is commonly associated with reduced treatment attendance that leads to variable treatment outcomes. ${ }^{87}$

Two recent topics have appeared in the voice literature, the concepts of coping and self-regulation, which are already acknowledged by psychologists dealing with behaviors. 


\section{Coping strategies}

Coping is defined as the manner with which an individual deals with a stressful situation. Once the individual faces a certain event that exceeds his/her adaptation resources, cognitive and behavioral efforts are used to manage either external or internal demands. ${ }^{88-93}$

Generally, coping strategies can be categorized as problem-focused and emotional-focused.$^{88}$ Some individuals will act directly into the stressful event using cognitive strategies to modify the situation, while others will use emotional strategies to alleviate its psychological consequences. The function of coping is to promote the adaptation of the individual to the unsettling situation. The sense of how much control the individual perceives to have over the situation will in a way define the coping to be used. ${ }^{89-91,94-96}$ Consequently, when a health problem is associated with controllable aspects, patients tend to engage practical solutions directed to the problem itself. On the other hand, when the illness is not curable and related factors cannot be controlled, people tend to utilize strategies to manage emotions. ${ }^{97,98}$

When the mediating role of coping is taken into consideration, ${ }^{89-91}$ it becomes easier to understand the diversity of the treatment outcome of a voice problem and the importance of addressing adequately this issue during both the evaluation and intervention processes, since the expected result is the effective adaptation of the individual to the situation. Speechlanguage pathologists should help patients identify the strategies they are using to cope with their voice disorder and to assist them toward changing maladaptive strategies. ${ }^{99,100}$ During the evaluation session, the clinician should make use of a specific coping self-assessment tool to guide them in identifying and listing the nonadequate strategies. Over the course of therapy, the patient should also be encouraged to use problem-adequate strategies. ${ }^{101}$

The first researchers who investigated coping strategies with dysphonia looked at individuals with spasmodic dysphonia and with MTD using the Voice Disability Coping Questionnaire. ${ }^{102}$ The results showed that individuals with spasmodic dysphonia used more emotion-focused strategies. The same questionnaire was used to investigate a Brazilian population with and without vocal complaints; overall, individuals with vocal complaints use several different strategies to cope with their voice problem, especially problem-focused strategies. ${ }^{101}$ Teachers with and without vocal complaint that sought (FD cases) or did not seek professional help were also studied. ${ }^{103}$ The interesting conclusion was that teachers who had a voice disorder and looked for help used more coping strategies when compared to the other two groups.
In accordance with the previous Brazilian study ${ }^{101}$ the teachers also tended to use more problem-focused strategies. ${ }^{103}$

An important aspect that needs to be considered when studying coping is the role of culture. Within a specific culture, certain types of coping strategies will be more or less effective in fostering emotional well-being and in addressing problems that cause stress. ${ }^{104}$ The information about the role of culture on coping strategies can also be taken into consideration when designing interventions, especially for culturally diverse populations. The literature about the topic does not have multicentric studies that include samples from different countries; however, there are studies that compare ethnic groups within a certain society. ${ }^{105,106}$ These studies have distinct designs and utilize diverse assessment instruments; hence the findings obtained are manifold. For this reason, the results many times cannot be compared. In the field of voice, there are no studies that compare the influence of culture on coping with voice problem. Consequently, further research should include a multicultural population in order to investigate the effect of culture on coping with voice problems.

\section{Self-regulation strategies}

Self-regulation, as well as coping strategies, may have been underestimated in patients with FD. Perceived control is a central construct in psychology. Present perceived control (PPC), opposed to control in the past, is the perception of having some kind of control over some current aspect of the event. ${ }^{107}$ It is especially important when there are stressful life situations, such as a voice disorder limiting the ability to communicate socially or at work. PPC is important in adjustment to stress and to help the clinician facilitate patient's control over his/her voice. Present control is related to lower perceived distress, which also benefits the patient's improvement during vocal rehabilitation.

On a large study exploring the relation among distress, stress, vocal handicap and perceived control, ${ }^{108}$ authors concluded that vocal handicap was more related to distress among those individuals with low perceived control. The severity of distress and vocal handicap were positively correlated, and the relation between them was moderated by perceived control. The authors used PPC subscale, with eight items (from a total of 17 sentences scale). These were the items most strongly associated with outcomes, adapted to be used in the context of a voice problem. ${ }^{108}$ The authors reinforce the fact that the present control reflects the perceived ability to control one's reaction to a stressful event and it is not the same as coping. Data from the general population on stressful events have shown that the avoidant coping could 
be related to more distress, ${ }^{107}$ but this was not investigated in dysphonic patients. If PPC is related to outcomes, it is still a question to be determined.

\section{Voice rehabilitation}

Vocal rehabilitation consists of direct and indirect approaches to ameliorate voice problems. Vocal rehabilitation is the primary management choice for FD treatment. An indirect approach includes education about voice and communication, vocal health information, as well as counseling regarding stress management and relaxation. On the other hand, direct therapy consists of specific exercises to control and coordinate the different aspects of the voice production, based on the large information obtained from the multidimensional evaluation. ${ }^{109}$ A method of rehabilitation described since the $1990 \mathrm{~s}$ is the perilaryngeal manipulation. ${ }^{110}$ There are several different approaches of laryngeal manipulation ${ }^{4,10,111}$ and evidence of its positive results, regardless of the type of manipulation used, particularly for MTD cases. ${ }^{10}$

The challenge for the speech-language pathologist is to obtain the most accurate diagnosis and to select an effective program of treatment for a specific patient with FD. Even if we admit that FD patients may present with related psychodynamic issues, when FD is used as a synonym of MTD, muscle activity is the main feature that should be addressed clinically. ${ }^{4,10}$ Before starting treatment, the first session is usually performed to confirm the diagnosis. During this session, diagnostic probes or muscular palpation are tried out. ${ }^{9}$ The probes may include manual palpation of the extrinsic muscles of the larynx, nonspeech tasks, visual and audio instrumental feedback, inhalatory phonation, lip trills, task-specific sentences to distinguish MTD from spasmodic dysphonia, and perceptual and compensatory behavior assessments.

Vocal rehabilitation is typically administered worldwide once or twice a week, in sessions of 30 minutes -45 minutes, delivered by a single clinician. ${ }^{18,21}$ This format enhances progressive learning, favors patient-clinician rapport and seems to be ideal for sustained behavioral changes. Cognitive behavioral therapy also appears to be an additional effective approach in the treatment of FD, by reducing associated distress. ${ }^{112}$ However, in cases of FD due to MTD, usually associated with complete aphonia or whispered phonation, voice is restored in few sessions of intensive laryngeal manipulation treatment. ${ }^{4}$

The ultimate goal of vocal rehabilitation is to restore normal voice. There are few randomized clinical trials that investigated patients with FD. One study compared two different therapeutic programs for the treatment of behavioral voice problem (FD was referred to as a behavioral problem and not as MTD). The treatment options were the Vocal Function Exercises and the Comprehensive Program for Voice Rehabilitation. In addition to these two therapeutic methods, the authors administered a vocal hygiene session. They concluded that both options offer good results, with positive outcomes in the laryngeal visual examination, perceptual and acoustical analysis as well as in the selfassessment scales. ${ }^{14}$ Although indirect and direct combined intervention approaches seem to be more efficient, ${ }^{113,114}$ the method chosen to deliver voice therapy should be based on the clinician's or patient's preferences or even on the need to include global communications aspects, particularly for professional voice users. ${ }^{14}$

A visual examination of the larynx can be used as a direct feedback tool during vocal rehabilitation, regardless of the fact that stroboscopic findings do not always correspond with voice improvement. ${ }^{24}$ It allows patients to realize what the mechanics of voice is and how the treatment affected the laryngeal configuration and activity. ${ }^{115}$

Vocal rehabilitation in FD is effective; ${ }^{116}$ however, traditional voice therapy can be unsuccessful for some patients, often associated with poor compliance or lack of adherence to sessions and treatment. ${ }^{117-119}$ This fact may lead clinicians to frustration. ${ }^{120}$ An alternative approach is to offer intensive programs, particularly in cases of recalcitrant dysphonias, with previous treatment failures. ${ }^{117}$ The intensive regimen is based on motor learning theory, neurobiology, exercise physiology, and psychotherapy. This approach also permits customization in order to fulfill the patient's demands and to assist in the transferring of acquired skills into spontaneous speech. ${ }^{121}$ Not only from a clinician's perception, but also from a patient's perspective, intensive treatment achieves a high level of satisfaction with vocal therapy and reduced voice handicap after treatment. ${ }^{87}$ In addition, it has been considered as one of the best ways to improve client adherence and treatment outcomes. ${ }^{87,117,119}$ The results achieved by intensive therapy in 1 day may correspond to a 2-week regular regimen. ${ }^{117}$ There are no controlled studies comparing regular vs intensive approaches for FD and, of course, overdoses of exercises and training should be taken into consideration. ${ }^{122}$

Patients are usually asked to perform exercises out of the therapy session considering that functional cortical reorganization depends on specific training. ${ }^{123}$ Daily practice allows voice stabilization and promotes continued improvement in vocal quality, acoustic measures, self-assessment, and 
other outcome measures. ${ }^{109}$ Conventional voice therapy and laryngeal manipulation have shown a moderate treatment effect. Nonetheless, a customized approach to the patient's limitations and the lack of control groups in many studies restrict the quality of evidence. ${ }^{109}$

There is an increasing understanding of the need to employ high-quality outcome measures in clinical research. For FD cases, the difficulty is even greater and starts with a basic problem, such as, terminology. Other problems include the need of a multidimensional approach for diagnosis, the lack of a single characteristic vocal quality related to this disorder, comprehension of differential diagnosis, and the influence of psychological aspects. The last problem would require a tailored approach to the patient's treatment.

\section{Strategies for improving patient outcome}

Health-related quality of life is a broad concept that refers to patient-perceived impact of the disease and treatment on physical, psychological, and social function (World Health Organization). ${ }^{78}$ The individual's well-being is the core of this concept. Patient-related outcomes were initially defined as subjective health indicators that allow disability and illness to be assessed, based on the patient, caregiver, or physician self-reports. ${ }^{124}$ Patients' opinion should be considered at any decision-making level, including evaluation and treatment. The main tools for comprehending and measuring the consequences of health disorders are self-rating questionnaires that reflect a direct patient-reporting method. If this is carried out with well-constructed instruments, it can become a robust platform to implement and sustain public health strategies. ${ }^{125}$

Strategies to improve the FD patient outcome involve a series of procedures, including (Figure 1) the following: 1) proper diagnosis to exclude neurological and psychiatric disorders that can have similar physical presentation and can require the use of vocal probes for differential diagnosis; 2) careful recording of the voice signal with quantitative measurement and qualitative description of the vocal deviation for comparison after treatment; 3) acoustic evaluation including both extraction of selected parameters and description of the spectrographic trace, to gather data on the mechanism involved in voice production; 4) self-assessment questionnaires to map the impact of the voice problem and to comprehend the dimensions involved; 5) referral to a psychological evaluation in cases of suspected anxiety and/or depression; 6) identification of coping strategies to face dysfunctional approaches; 7) self-regulation data to assist the patient regarding vocal load; and finally 8) direct and intensive vocal rehabilitation to reduce psychological resistance and to reassure patients recovery.

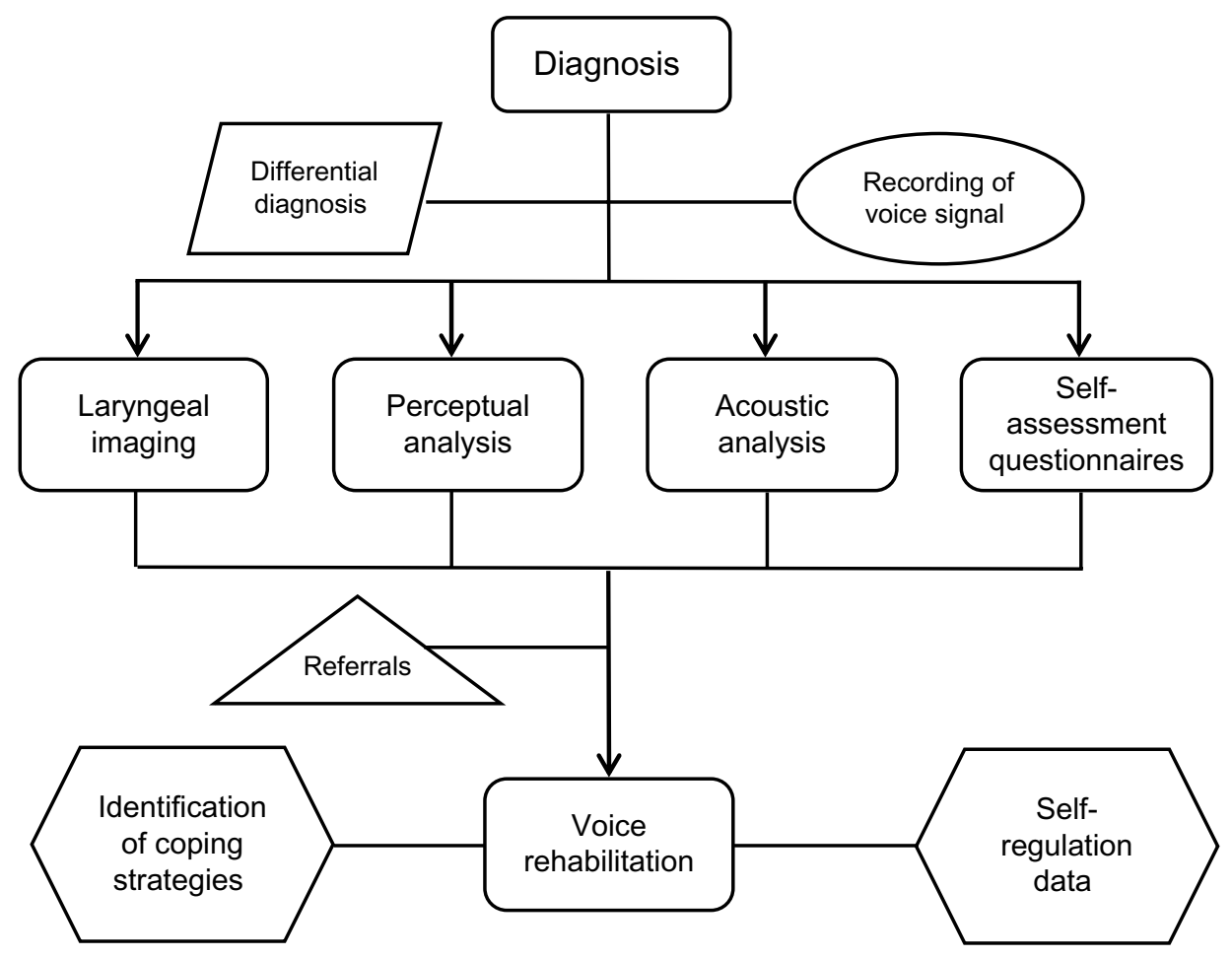

Figure I Flowchart with strategies to improve FD patient outcome. Abbreviation: FD, functional dysphonia. 


\section{Disclosure}

The authors report no conflicts of interest in this work.

\section{References}

1. Schwartz SR, Cohen SM, Dailey SH, et al. Clinical practice guideline: hoarseness (dysphonia). Otolaryngol Head Neck Surg. 2009;141(3):S1-S31.

2. Behlau M, Pontes P. Avaliação e Tratamento das Disfonias [Assessment and Treatment of Dysphonias]. São Paulo: Lovise; 1995:17-37. Portuguese.

3. Perelló J. Disfonies fonctionnelles. Folia Phoniatr. 1962;14:150-205.

4. Roy N, Bless DM, Heisey D, Ford CN. Manual circumlaryngeal therapy for functional dysphonia: an evaluation of short- and long-term treatment outcomes. J Voice. 1997;11:321-331.

5. Roy N, Bless DM, Heisey D. Personality and voice disorders: a multitrait-multidisorder analysis. J Voice. 2000;14(4):521-548.

6. Verdolini K, Rosen C, Branski R. Classification Manual for Voice Disorders - I. Special Interest Division 3, Voice and Voice Disorders. Rockville: ASHA; 2006:249-256.

7. Butcher P, Elias A, Cavali L. Understanding and Treating Psychogenic Voice Disorder: A Cognitive Behavioral Therapy Framework. Chichester: Wiley; 2007.

8. Van Houtte E, Van Lierde K, Claeys S. Pathophysiology and treatment of muscle tension dysphonia: a review of the current knowledge. JVoice. 2011;25(2):202-207.

9. Hancock R. Management of functional aphonia and related severe muscle tension disorders: a practical approach with historical underpinnings. Perspect Voice Voice Disord. 2014;24(2):55-63.

10. Mathieson L. The evidence for laryngeal manual therapies in the treatment of muscle tension dysphonia. Curr Opin Otolaryngol Head Neck Surg. 2011;19(3):171-176.

11. Pontes P, Behlau M, Kyrillos L. Glottic configurations and glottic proportion: an attempt to understand the posterior glottic chink. Rev Otol Rhinol Laryngol. 1994;115:261-266.

12. Dietrich M, Verdolini Abbott K, Gartner-Schmidt J, Rosen CA. The frequency of perceived stress, anxiety, and depression in patients with common pathologies affecting voice. $J$ Voice. 2008;22(4):472-488.

13. Siupsinskiene N, Razbadauskas A, Dubosas L. Psychological distress in patients with benign voice disorders. Folia Phoniatr Logop. 2011;63(6): 281-288.

14. Pedrosa V, Pontes A, Pontes P, Behlau M, Peccin SM. The effectiveness of the comprehensive voice rehabilitation program compared with the vocal function exercises method in behavioral dysphonia: a randomized clinical trial. J Voice. 2015. Epub ahead of print.

15. Litts JK, Gartner-Schmidt JL, Clary MS, Gillespie AI. Impact of laryngologist and speech pathologist coassessment on outcomes and billing revenue. Laryngoscope. 2015;125(9):2139-2142.

16. Jacobson BH, Johson A, Grywalsky C, et al. The voice handicap index (VHI): development and validation. Am J Speech Lang Pathol. 1997;6: $66-70$.

17. Hogikyan ND, Sethuraman G. Validation of an instrument to measure voice- related quality of life (V-RQOL). J Voice. 1999;13:557-569.

18. Carding PN, Horsley IA, Docherty GJ. A study of the effectiveness of voice therapy in the treatment of 45 patients with non-organic dysphonia. J Voice. 1999;13(1):72-104.

19. Ma EP, Yiu EM. Voice activity and participation profile: assessing the impact of voice disorders on daily activities. J Speech Lang Hear Res. 2001;44(3):511-524.

20. Deary IJ, Wilson JA, Carding PN, MacKenzie K. VoiSS: a patient-derived voice symptom scale. J Psychosom Res. 2003;54(5):483-489.

21. Behlau M, Murry T. International and intercultural aspects of voice and voice disorders. In: Battle DE, editor. Communication Disorders in Multicultural and International Populations. 4th ed. St Louis, MO: Elsevier; 2012:174-207.

22. Mehta DD, Hillman RE. Current role of stroboscopy in laryngeal imaging. Curr Opin Otolaryngol Head Neck Surg. 2012;20(6):429-436.
23. Paul BC, Chen S, Sridharan S, Fang Y, Amin MR, Branski RC. Diagnostic accuracy of history, laryngoscopy, and stroboscopy. Laryngoscope. 2013;123(1):215-219.

24. Halawa WE, Muñoz IV, Perez SS. Effectiveness of laryngostroboscopy for monitoring the evolution of functional dysphonia after rehabilitator treatment. Indian J Otolaryngol Head Neck Surg. 2013;65(4): 322-326.

25. Oates J. Auditory-perceptual evaluation of disordered vocal quality pros, cons and future directions. Folia Phoniatr Logop. 2009;61(1): $49-56$.

26. Kreiman J, Gerratt B, Ito M. When and why listeners disagree in voice quality assessment tasks. J Am Stat Assoc. 2007;122(4):2354-2364.

27. Roy N. Optimal dose-response relationships in voice therapy. Int $J$ Speech Lang Pathol. 2012;14(5):419-423.

28. Hillman RE. SIGnatures: The Quest for Voice Assessment Guidelines: Special Interest Group 3, Voice and Voice Disorders. Rockville, MD: The ASHA Leader; 2013:54-55.

29. Patel S, Shrivastav R. Perception of dysphonic vocal quality: some thoughts and research update. Perspect Voice Voice Disord. 2007;17: 3-7.

30. Brockmann-Bauser M, Drinnan MJ. Routine acoustic voice analysis: time to think again. Curr Opin Otolaryngol Head Neck Surg. 2011;19: 165-170.

31. Rabinov CR, Kreiman J, Gerratt BR, Bielamowicz S. Comparing reliability of perceptual ratings of roughness and acoustic measures of jitter. J Speech Hear Res. 1995;38:26-32.

32. Titze IR. Workshop on Acoustic Voice Analysis: Summary Statement. National Center for Voice and Speech; Iowa City;1995.

33. Awan SN, Roy N, Zhang D. Validation of the cepstral spectral index of dysphonia (CSID) as a screening tool for voice disorders: development of clinical cutoff scores. J Voice. 2010;24(3):285-293.

34. Peterson EA, Roy N, Awan SN, Merrill RM, Banks R, Tanner K. Toward validation of the cepstral spectral index of dysphonia (CSID) as an objective treatment outcomes measure. J Voice. 2013;27(4):401-410.

35. Herzel D, Berry H, Titze IR, Saleh M. Analysis of vocal disorders with methods from nonlinear dynamics. J Speech Hear Res. 1994;37: 1008-1019.

36. Awan SN, Roy N, Jiang JJ. Nonlinear dynamic analysis of disordered voice: the relationship between the correlation dimension (D2) and pre-/post-treatment change in perceived dysphonia severity. $J$ Voice. 2010;24(3):285-293.

37. Nawka T, Wiesmann U, Gonnermann U. Validierung des voice handicap index (VHI) in der deutschen Fassung. HNO. 2003;51:921-930.

38. Hsiung MW, Lu P, Kang BH, Wang HW. Measurement and validation of the voice handicap index in voice-disordered patients in Taiwan. J Laryngol Otol. 2003;117(6):478-481.

39. Guimarães I, Abberton E. An investigation of the voice handicap index with speakers of Portuguese: preliminary data. J Voice. 2004;18(1): $71-82$.

40. Woisard V, Bodin S, Puech M. The voice handicap index: impact of the translation in French on the validation. Rev Laryngol Otol Rhinol (Bord). 2004;125(5):307-312.

41. Pruszewicz A, Obrebowski A, Wiskirska-Woznica B, Wojnowski W. Complex voice assessment-polish version of the voice handicap index (VHI). Otolaryngol Pol. 2004;58(3):547-549.

42. Franic DM, Bramlett RE, Bothe AC. Psychometric evaluation of disease specific quality of life instruments in voice disorders. $J$ Voice. 2005;19(2):300-315.

43. Günther S, Rasch T, Klotz M, Hoppe U, Eysholdt U, Rosanowski F. Bestimmung der subjektiven Beeinträchtigung durch Dysphonien. Ein Methodenvergleich. HNO. 2005;53:895-904.

44. Hakkesteegt MM, Wieringa MH, Gerritsma EJ, Feenstra L. Reproducibility of the Dutch version of the voice handicap index. Folia Phoniatr Logop. 2006;58(2):132-138.

45. Amir O, Ashkenazi O, Leibovitzh T, Michael O, Tavor Y, Wolf M. Applying the voice handicap index (VHI) to dysphonic and nondysphonic Hebrew speakers. $J$ Voice. 2006;20(2):318-324. 
46. Webb AL, Carding PN, Deary IJ, MacKenzie K, Steen IN, Wilson JA. Optimizing outcome assessment of voice interventions, I: reliability and validity of three self-reported scales. J Laryngol Otol. 2007;121(8): $763-767$.

47. Núñez-Batalla F, Corte-Santos P, Señaris-González B, LlorentePendás JL, Górriz-Gil C, Suárez-Nieto C. Adaptation and validation to the Spanish of the voice handicap index (VHI-30) and its shortened version (VHI-10). Acta Otorrinolaringol Esp. 2007;58(9):386-392.

48. Xu W, Li HY, Hu R, et al. Analysis of reliability and validity of the Chinese version of voice handicap index (VHI). Zhonghua Er Bi Yan Hou Tou Jing Wai Ke Za Zhi. 2008;43(9):670-675.

49. Kiliç MA, Okur E, Yildirim I, et al. Reliability and validity of the Turkish version of the voice handicap index. Kulak Burun Bogaz Ihtis Derg. 2008;18(3):139-147.

50. Ohlsson AC, Dotevall H. Voice handicap index in Swedish. Logoped Phoniatr Vocol. 2009;34(2):60-66.

51. Behlau M, Alves Dos Santos LM, Oliveira G. Cross-cultural adaptation and validation of the voice handicap index into Brazilian Portuguese. J Voice. 2011;25(3):354-359.

52. Schindler A, Ottaviani F, Mozzanica F, et al. Cross-cultural adaptation and validation of the voice handicap index into Italian. $J$ Voice. 2010 ; 24(6):708-714.

53. Helidoni ME, Murry T, Moschandreas J, Lionis C, PrintzaA, Velegrakis GA. Cross-cultural adaptation and validation of the voice handicap index into Greek. J Voice. 2010;24(2):221-227.

54. Malki KH, Mesallam TA, Farahat M, Bukhari M, Murry T. Validation and cultural modification of Arabic voice handicap index. Eur Arch Otorhinolaryngol. 2010;267(11):1743-1751.

55. Karlsen T, Grieg AR, Heimdal JH, Aarstad HJ. Cross-cultural adaption and translation of the voice handicap index into Norwegian. Folia Phoniatr Logop. 2012;64(5):234-240.

56. Taguchi A, Mise K, Nishikubo K, Hyodo M, Shiromoto O. Japanese version of voice handicap index for subjective evaluation of voice disorder. J Voice. 2012;26(5):.e15-.e19.

57. Moradi N, Pourshahbaz A, Soltani M, Javadipour S, Hashemi H, Soltaninejad N. Cross-cultural equivalence and evaluation of psychometric properties of voice handicap index into Persian. J Voice. 2013; 27(2):.e15-.e258

58. Bonetti A, Bonetti L. Cross-cultural adaptation and validation of the voice handicap index into croatian. J Voice. 2013;27(1):.e7-.e130.

59. Trinite B, Sokolovs J. Adaptation and validation of the voice handicap index in Latvian. $J$ Voice. 2014;28(4):452-457.

60. Rosen CA, Lee AS, Osborne J, Zullo T, Murry T. Development and validation of the voice handicap index-10. Laryngoscope. 2004;114(9): $1549-1556$

61. Lam PK, Chan KM, Ho WK, Kwong E, Yiu EM, Wei WI. Cross-cultural adaptation and validation of the Chinese voice handicap index-10. Laryngoscope. 2006;116(7):1192-1198.

62. Amir O, Tavor Y, Leibovitzh T, et al. Evaluating the validity of the voice handicap index-10 (VHI-10) among Hebrew speakers. Otolaryngol Head Neck Surg. 2006;135(4):603-607.

63. Costa T, Oliveira G, Behlau M. Validation of the voice handicap index: 10 (VHI-10) to the Brazilian Portuguese. Codas. 2013;25(5): $482-485$.

64. Forti S, Amico M, Zambarbieri A, et al. Validation of the Italian voice handicap index-10. J Voice. 2014;28(2):.e17-.e263.

65. Schwanfelder C, Eysholdt U, Rosanowski F, Graessel E. Voicerelated quality of life: structure, validity and factors of the German questionnaire. Folia Phoniatr Logop. 2008;60:241-248.

66. Gasparini G, Behlau M. Quality of life: validation of the Brazilian version of the voice-related quality of life (V-RQOL) measure. J Voice. 2009;23:76-81.

67. Deshpande MS, Kakade AC, Chaukar DA, et al. Validation and assessment of voice-related quality of life in Indian patients undergoing total laryngectomy and primary tracheoesophageal puncture. Head Neck. 2009;31(1):37-44.
68. Paulinelli BR, Gama ACC, Behlau M. Validation of the vocal performance questionnaire in Brazil. Rev Soc Bras Fonoaudiol. 2012;17(1): 85-91.

69. Sukanen O, Sihvo M, Rorarius E, Lehtihalmes M, Autio V, Kleemola L. Voice activity and participation profile (VAPP) in assessing the effects of voice disorders on patients' quality of life: validity and reliability of the Finnish version of VAPP. Logoped Phoniatr Vocol. 2007;32:3-8.

70. Ricarte A, Oliveira G, Behlau M. Validation of the voice activity and participation profile protocol in Brazil. Codas. 2013;25(3):242-249.

71. Fava G, Paolillo NP, Oliveira G, Behlau M. Cross-cultural adaptation, validation, and cutoff point of the Italian version of the voice activity and participation profile: Profilo di Attività e Partecipazione Vocale. J Voice. 2015;29(1):.e11-.e19.

72. Moreti F, Zambon F, Oliveira G, Behlau M. Cross-cultural adaptation, validation, and cutoff values of the Brazilian version of the voice symptom scale-VoiSS. J Voice. 2014;28(4):458-468.

73. Karnell MP, Melton SD, Childes JM, Coleman TC, Dailey SA, Hoffman HT. Reliability of clinician-based (GRBAS and CAPE-V) and patient-based (V-RQOL and IPVI) documentation of voice disorders. J Voice. 2007;21:576-590.

74. Ugulino AC, Oliveira G, Behlau M. Perceived dysphonia by the clinician's and patient's viewpoint. J Soc Bras Fonoaudiol. 2012;24(2): 113-118.

75. Krischke S, Weigelt S, Hoppe U, et al. Quality of life in dysphonic patients. J Voice. 2005;19(1):132-137.

76. Konnai RM, Jayaram M, Scherer RC. Development and validation of a voice disorder outcome profile for an Indian population. $J$ Voice. 2010;24(2):206-220.

77. Branski RC, Cukier-Blaj S, Pusic A, et al. Measuring quality of life in dysphonic patients: a systematic review of content development in patient-reported outcomes measures. J Voice. 2010;24:193-198.

78. World Health Organization. International Classification of Functioning, Disability, and Health. Geneva, Switzerland: World Health Organization; 2001.

79. Gliklich RE, Glovsky RM, Montgomery WW. Validation of a voice outcome survey for unilateral vocal cord paralysis. Otolaryngol Head Neck Surg. 1999;120:153-158.

80. Cohen SM, Jacobson BH, Garrett CG, et al. Creation and validation of the singing voice handicap index. Ann Otol Rhinol Laryngol. 2007;116(6):402-406.

81. Murry T, Zschommler A, Prokop J. Voice handicap in singers. J Voice. 2009;23:376-379.

82. Nguyen DD, Kenny DT, Tran ND, Livesey JR. Muscle tension dysphonia in Vietnamese female teachers. J Voice. 2009;23(2):195-208.

83. Leonard KL. Is patient satisfaction sensitive to changes in the quality of care? An exploitation of the Hawthorne effect. J Health Econ. 2007; 27(2):444-459.

84. Freidl W, Friedrich G, Egger J, Fitzek T. Psychogenic aspects of functional dysphonia. Folia Phoniatr. 1993;45(1):10-13.

85. Deary V, Miller T. Reconsidering the role of psychosocial factors in functional dysphonia. Curr Opin Otolaryngol Head Neck Surg. 2011; 19(3):150-154.

86. Kiese-Himmel C. Clinical-psychological components in the consideration of functional dysphonia-a review. Laryngorhinootologie. 2015; 94(3):156-162.

87. Wenke RJ, Stabler P, Walton C, et al. Is more intensive better? Client and service provider outcomes for intensive versus standard therapy schedules for functional voice disorders. $J$ Voice. 2014;28(5): .e31-.e652.

88. Folkman S. Stress, Appraisal and Coping. New York, NY: Springer Publishing Company; 1984

89. Folkman S. Personal control and stress and coping processes: a theoretical analysis. J Pers Soc Psychol. 1984;46:839-852.

90. Folkman S, Lazarus RS. If it changes it must be a process: study of emotion and coping during three stages of a college examination. J Pers Soc Psychol. 1985;48:150-170. 
91. Folkman S, Lazarus RS, Dunkel-Schetter C, DeLongis A, Gruen RJ. Dynamics of a stressful encounter: cognitive appraisal, coping, and encounter outcomes. J Pers Soc Psychol. 1986;50:992-1003.

92. Lazarus RS. Coping theory and research: past, present, and future. Psychosom Med. 1993;55:234-247.

93. Lazarus RS. Fifty Years of the Research and Theory of RS Lazarus: An Analysis of Historical and Perennial Issues. Mahwah, NJ: Lawrence Erlbaum Associates; 1998.

94. Holahan CJ, Moos RH. Personal and contextual determinants of coping strategies. J Pers Soc Psychol. 1987;52:946-955.

95. Carver CS, Scheier MF, Weintraub JK. Assessing coping strategies: a theoretically based approach. J Pers Soc Psychol. 1989;56: 267-283.

96. David JP, Suls J. Coping efforts in daily life: role of big five traits and problem appraisals. J Pers. 1999;67:265-294.

97. Chesney MA, Chambers DB, Taylor JM, Johnson LM, Folkman S. Coping effectiveness training for men living with HIV: results from a randomized clinical trial testing a group-based intervention. Psychosom Med. 2003;65:1038-1046.

98. Hagger MS, Orbell S. A meta-analytic review of the common-sense model of illness representations. Psychol Health. 2003;18:141-184.

99. Van Opstal MJMC. A systematic, holistic and integrative process of self-control for voicing with optimal coping effects in teachers 2. A process of change - an expert's opinion. Folia Phoniatr Logop 2010;62:71-85.

100. Meulenbroek LF, de Jong FI. Trainee experience in relation to voice handicap, general coping and psychosomatic well-being in female student teachers: a descriptive study. Folia Phoniatr Logop. 2010;62: $47-54$.

101. Oliveira G, Hirani SP, Epstein R, Yazigi L, Behlau M. Coping strategies in voice disorders of a Brazilian population. J Voice. 2012;26(2): 205-213.

102. Epstein R, Hirani SP, Stygall J, Newman SP. How do individuals cope with voice disorders? introducing the voice disability coping questionnaire. $J$ Voice. 2009;23:209-217.

103. Zambon F, Moreti F, Behlau M. Coping strategies in teachers with vocal complaint. J Voice. 2014;28(3):341-348.

104. Folkman S, Moskowitz JT. Coping: pitfalls and promise. Ann Rev Psychol. 2004;55:745-774.

105. Anderson M, Elam G, Solarin I, Gerver S, Fenton K, Easterbrook P. Coping with HIV: Caribbean people in the United Kingdom. Qual Health Res. 2009;19:1060-1075.

106. Côté J, Delmas P, Delpierre C, Sylvain H, Delon S, Rouleau G. Factors related to quality of life in treatment-adherence, successfully treated HIV patients in France. Open Nurs J. 2009;3:10-17.

107. Frazier P, Keenan N, Anders S, Perera S, Shallcross S, Hintz S. Perceived past, present, and future control and adjustment to stressful life events. J Pers Soc Psychol. 2011;100(4):749-765.

108. Misono S, Meredith L, Peterson CB, Frazier PA. New perspective on psychosocial distress in patients with dysphonia: the moderating role of perceived control. $J$ Voice. 2015. Epub ahead of print.
109. Bos-Clark M, Carding P. Effectiveness of voice therapy in functional dysphonia: where are we now? Curr Opin Otolaryngol Head Neck Surg. 2011;19:160-164.

110. Aronson AE. Clinical Voice Disorders: An Interdisciplinary Approach. New York, NY: Thieme; 1980.

111. Mathieson L, Hirani SP, Epstein R, Baken RJ, Wood G, Rubin JS. Laryngeal manual therapy: a preliminary study to examine its treatment effects in the management of muscle tension dysphonia. J Voice. 2009;3:353-366.

112. Daniilidou P, Carding P, Wilson J, Drinnan M, Deary V. Cognitive behavioral therapy for functional dysphonia: a pilot study. Ann Otol Rhinol Laryngol. 2007;116(10):17-122.

113. Niebudek-Bogusz E, Sznurowska-Przygocka B, Fiszer M, et al. The effectiveness of voice therapy for teachers with dysphonia. Folia Phoniatr Logop. 2008;60:134-141.

114. Morsomme D, de la Bardonnie MF, Verduyckt I, Jamart J, Remacle M. Subjective evaluation of the long-term efficacy of speech therapy on dysfunctional dysphonia. $J$ Voice. 2010;24:178-182.

115. Rattenbury HJ, Carding PN, Finn P. Evaluating the effectiveness and efficiency of voice therapy using transnasal flexible laryngoscopy: a randomized controlled trial. J Voice. 2004;18:522-533.

116. Ruotsalainen J, Sellman J, Lic P, Lehto L. Systematic review of the treatment of functional dysphonia and prevention of voice disorders. Otolaryngol Head Neck Surg. 2008;138:557-565.

117. Behlau M, Madazio G, Pacheco C, Gielow I. Intensive short-term voice therapy: the Brazilian experience. Perspect Voice Voice Disord. 2014;24:98-103.

118. Portone C, Johns MM, Hapner ER. A review of patient adherence to the recommendation for voice therapy. J Voice. 2008;22:192-196.

119. van Leer E, Connor NP. Patient perceptions of voice therapy adherence. J Voice. 2010;24(4):458-469.

120. Bogaardt H, Hakkesteegt M, Grolman W, Lindeboom R. Validation of the voice handicap index using Rasch analysis. $J$ Voice. 2007;21: 337-344

121. Patel RR, Bless DM, Thibeault SL. Boot camp: a novel intensive approach to voice therapy. J Voice. 2011;25:562-569.

122. Roy N, Barkmeier-Kraemer J, Eadie T, et al. Evidence-based clinical voice assessment: a systematic review. Am J Speech Lang Pathol. 2013;22(2):212-226.

123. Hamzei F, Liepert J, Dettmers C, Weiller C, Rijntjes M. Two different reorganization patterns after rehabilitative therapy: an exploratory study with FMRI and TMS. Neuroimage. 2006;31:710-720.

124. Acquardo C, Berzon R, Dubois D, et al. Incorporating the patient's perspective into drug development and communication: an ad hoc task force report of the patient-reported outcomes (PRO) Harmonization Group meeting at the Food and Drug Administration. 2001. Value Health. 2003;6:522-531.

125. Farnik M, Pierzchała W. Instrument development and evaluation for patient-related outcomes assessments. Patient Relat Outcome Meas. 2012;3:1-7.
Patient Related Outcome Measures

\section{Publish your work in this journal}

Patient Related Outcome Measures is an international, peer-reviewed, open access journal focusing on treatment outcomes specifically relevant to patients. All aspects of patient care are addressed within the journal and practitioners from all disciplines are invited to submit their work as well as healthcare researchers and patient support groups.

\section{Dovepress}

The manuscript management system is completely online and includes a very quick and fair peer-review system. Visit http://www.dovepress. com/testimonials.php to read real quotes from published authors. 